This item was submitted to Loughborough's Research Repository by the author.

Items in Figshare are protected by copyright, with all rights reserved, unless otherwise indicated.

\title{
Resource provisioning in wireless virtualized networks via massive-MIMO
}

PLEASE CITE THE PUBLISHED VERSION

http://dx.doi.org/10.1109/LWC.2015.2402126

\section{PUBLISHER}

(c) IEEE

\section{VERSION}

AM (Accepted Manuscript)

\section{PUBLISHER STATEMENT}

This work is made available according to the conditions of the Creative Commons Attribution-NonCommercialNoDerivatives 4.0 International (CC BY-NC-ND 4.0) licence. Full details of this licence are available at: https://creativecommons.org/licenses/by-nc-nd/4.0/

\section{LICENCE}

CC BY-NC-ND 4.0

\section{REPOSITORY RECORD}

Jumba, Vikas, Saeedeh Parsaeefard, Mahsa Derakhshani, and Tho Le-Ngoc. 2019. "Resource Provisioning in Wireless Virtualized Networks via Massive-mimo". figshare. https://hdl.handle.net/2134/21229. 


\title{
Resource Provisioning in Wireless Virtualized Networks via Massive-MIMO
}

\author{
Vikas Jumba*, Saeedeh Parsaeefard*, Mahsa Derakhshani ${ }^{\dagger}$, Tho Le-Ngoc* \\ ${ }^{*}$ Department of Electrical \& Computer Engineering, McGill University, Montreal, QC, Canada \\ ${ }^{\dagger}$ Department of Electrical \& Computer Engineering, University of Toronto, Toronto, ON, Canada \\ Email: vikas.jumba@mail.mcgill.ca; saeideh.parsaeifard@mcgill.ca; mahsa.derakhshani@utoronto.ca; tho.le-ngoc@mcgill.ca
}

\begin{abstract}
This letter proposes a dynamic resource provisioning scheme for an OFDMA wireless virtualized network (WVN), where one base-station equipped with a large number of antennas serves users belonging to a number of service providers via different slices. In particular, joint power, sub-carrier, and antenna allocation problems are presented for both perfect and imperfect channel knowledge cases, aiming to maximize a sum-utility while maintaining a minimum rate per slice. Subsequently, relaxation and variable transformation are applied to develop the efficient algorithm to solve the formulated non-convex, combinational optimization problem. Simulation results reveal the benefits of applying a large number of antennas in this setup and evaluate the network performance for different system conditions.
\end{abstract}

Index Terms-Large number of antennas, resource provisioning, wireless network virtualization.

\section{INTRODUCTION}

Wireless network virtualization is a promising approach to provide service customization for next-generation wireless networks. In wireless virtualized networks (WVNs), limited wireless resources (i.e., power and spectrum) are shared among different groups of users referred to as slices. Each slice may have a different set of quality-of-service (QoS) requirements, which calls for effective resource provisioning algorithms in WVNs to maximize the sum utility of all slices, while holding the minimum required rate of each slice [1].

However, due to the random nature of wireless channels and, power and spectrum limitations, there is always a non-zero probability that the minimum required rate cannot be satisfied. Thus, this will cause infeasibility problem for resource provisioning and lead to a poor network performance. In this paper, to expand the feasibility region, we take advantage of the large spatial degree of freedom introduced by massive multi-inputmulti-output (MIMO) technology. In massive MIMO, each base station (BS) is equipped with a large number of antennas serving single-antenna multiple users.

Developing the massive-MIMO scales up the MIMO multiplexing gain provided that the exact channel state information (CSI) of all users are available at BS [2]. However, extracting the precise values of CSI requires ideal orthogonal pilot signals between different BSs which is practically infeasible [2]. In this paper, taking this issue into account, we consider two cases: 1) perfect CSI where it is ideally assumed to have all the precise values of CSI; 2) imperfect CSI where the effect of non-orthogonal pilots is considered in the achievable rate.

Subsequently, we formulate a resource provisioning problem for the up-link transmission in a WVN. The objective is to maximize the sum utility of slices subject to the minimum required rate of each slice and transmit power of each user within each slice. We introduce a new utility function representing the difference between the total achieved user rate and its corresponding costs for allocated power and antennas. Considering two different pricing mechanisms for the allocated power and antennas enables effective control of inter-slice interference and available antennas in WVN. To the best of our knowledge, there exists no related work in the context of WVN with massive MIMO.

As the formulated problem is both non-convex and combinatorial, it suffers from high computational complexity. To develop an efficient algorithm, we apply variable transformations and constraint relaxations. Simulation results reveal that the values of power and antenna pricing variables have a significant impact on the total achieved user throughput.

The rest of this paper is organized as follows. Section II introduces the system model and problem formulations. Section III provides a solution for the formulated resource provisioning problem followed by Section V where the simulation results are presented. Section VI provides concluding remarks.

\section{Network Model and Problem Formulation}

We consider the up-link transmission in an orthogonal frequency division multiple access (OFDMA) WVN where a BS with $M$ antennas serves a set of slices $\mathcal{G}=\{1, \cdots, G\}$. Each slice $g \in \mathcal{G}$ has a set of single-antenna users denoted by $\mathcal{N}_{g}=\left\{1, \cdots, N_{g}\right\}$ and requires a minimum rate $R_{g}^{\text {rsv }}$. It should be noted that $N=\sum_{g \in \mathcal{G}} N_{g}$ and $N \ll M$. Consider $\mathbf{M}=\left[\mathbf{M}_{1}, \cdots, \mathbf{M}_{G}\right]$ as the allocated antenna vector for all slices where $\mathbf{M}_{g}=\left[\mathbf{M}_{n_{g}}\right]_{n_{g}=1}^{N_{g}}, \mathbf{M}_{n_{g}}=\left[M_{n_{g}, 1}, \cdots, M_{n_{g}, K}\right]$, and $M_{n_{g}, k}$ is the number of antennas allocated to user $n_{g}$ on sub-carrier $k$. Let $\mathbf{w}=\left[\mathbf{w}_{1}, \cdots, \mathbf{w}_{G}\right]$ be the sub-carrier assignment vector for all slices where $\mathbf{w}_{g}=\left[\mathbf{w}_{n_{g}}\right]_{n_{g}=1}^{N_{g}}$, $\mathbf{w}_{n_{g}}=\left[w_{n_{g}, 1}, \cdots, w_{n_{g}, K}\right]$, and $w_{n_{g}, k}=1$ indicates that subcarrier $k$ is allocated to user $n_{g}$ and otherwise $w_{n_{g}, k}=0$.

In this setup, let $\mathbf{h}_{n_{g}, k} \in \mathbb{C}^{1 \times M_{n_{g}, k}}$ be the channel vector of user $n_{g}$ on sub-carrier $k$, where $h_{n_{g}, k, m_{n_{g}, k}}$ is the channel coefficient of user $n_{g}$ on sub-carrier $k$ and antenna $m_{n_{g}, k}$. More specifically, $h_{n_{g}, k, m_{n_{g}, k}}=g_{n_{g}, k, m_{n_{g}, k}} \sqrt{d_{n_{g}}}$ where $g_{n_{g}, k, m_{n_{g}, k}}$ represents the small-scale fading coefficient with variance of 1 , and $d_{n_{g}}$ denotes the large-scale fading coefficient of user $n_{g}$ on sub-carrier $k$. Note that $d_{n_{g}}$ includes both path loss and shadowing [2]. Practically, the channel 
coefficients are estimated by the BS based on the uplink pilots with duration $\tau$ at the specific part of the coherence interval of $T$ [3]. In the perfect CSI case, the up-link received sample at BS after using the linear detector from user $n_{g} \in \mathcal{N}_{g}$ on sub-carrier $k$ is [3]

$$
y_{n_{g}, k}^{\text {Perf }}=\sqrt{P_{n_{g}, k}} \mathbf{h}_{n_{g}, k} \mathbf{f}_{n_{g}, k}^{\text {Perf }} x_{n_{g}, k}+I_{n_{g}, k}+\mathbf{z}_{n_{g}, k} \mathbf{f}_{n_{g}, k}^{\text {Perf }},
$$

where $x_{n_{g}, k}$ and $\mathbf{f}_{n_{g}, k}^{\text {Perf }} \in \mathbb{C}^{M_{n_{g}, k} \times 1}$ represent the transmit symbol and the precoding vector of user $n_{g}$ on sub-carrier $k$, respectively. Moreover, $P_{n_{g}, k}$ is the transmit power of user $n_{g}$ on sub-carrier $k$ and $\mathbf{z}_{n_{g}, k}$ is a vector of additive white Gaussian noise (AWGN) at the BS with zero mean and power spectral density $\sigma$, which is assumed to be independent and identically distributed (i.i.d.) for all users over all sub-carriers. For notational simplicity, we normalize $\sigma$ to 1 and thus $P_{n_{g}, k}$ is the transmit SNR and dimensionless. $I_{n_{g}, k}=\sum_{g \in \mathcal{G}} \sum_{n^{\prime} g \neq n_{g}} \sqrt{P_{n_{g}^{\prime}, k}} \mathbf{h}_{n_{g}^{\prime}, k} \mathbf{f}_{n_{g}, k}^{\text {Perf }} x_{n_{g}^{\prime}, k}$ is the interference from other users to user $n_{g}$. In the imperfect CSI case, due to pilot contamination error in the channel estimation and the linear detector, the received signal from user $n_{g}$ on subcarrier $k$ is [3]

$$
y_{n_{g}, k}^{\text {Imperf }}=\sqrt{P_{n_{g}, k}} \widetilde{\mathbf{h}}_{n_{g}, k} \mathbf{f}_{n_{g}, k}^{\text {Imperf }} x_{n_{g}, k}+\delta_{n_{g}, k}(\mathbf{P})
$$

where $\widetilde{\mathbf{h}}_{n_{g}, k}$ is the estimated channel vector including the contamination error, $\mathbf{P}=\left[\mathbf{P}_{1}, \cdots, \mathbf{P}_{G}\right]$ is the allocated power vector of all slices in which $\mathbf{P}_{g}=\left[\mathbf{P}_{n_{g}}\right]_{n_{g}=1}^{N_{g}}$ and $\mathbf{P}_{n_{g}}=$ $\left[P_{n_{g}, 1}, \cdots, P_{n_{g}, K}\right], \mathbf{f}_{n_{g}, k}^{\text {Imperf }} \in \mathbb{C}^{M_{n_{g}, k} \times 1}$ is the precoding vector in the imperfect CSI case, and $\delta_{n_{g}, k}(\mathbf{P})$ is the function of contamination error, interference and noise (See Appendix).Using maximum ratio combining (MRC) detector with $M \rightarrow \infty$, the rate of user $n_{g}$ on sub-carrier $k$ is derived in Appendix as

$R_{n_{g}, k}=$

$\left\{\begin{array}{l}\log _{2}\left(1+P_{n_{g}, k} d_{n_{g}} M_{n_{g}, k}\right), \\ \frac{T-\tau}{T} \log _{2}\left(1+\tau P_{n_{g}, k}^{2} d_{n_{g}}^{2} M_{n_{g}, k}\right)\end{array}\right.$,

for perfect CSI, for imperfect CSI,

and the total achieved rate of user $n_{g}$ is $R_{n_{g}}(\mathbf{P}, \mathbf{w}, \mathbf{M})=$ $\sum_{k \in \mathcal{K}} w_{n_{g}, k} R_{n_{g}, k}$. Now, we define utility function of slice $g$ as

$$
\begin{aligned}
& U_{g}(\mathbf{P}, \mathbf{w}, \mathbf{M})=\sum_{n_{g} \in \mathcal{N}_{g}} R_{n_{g}}(\mathbf{P}, \mathbf{w}, \mathbf{M})- \\
& \quad c_{g}^{\mathrm{M}} \sum_{n_{g} \in \mathcal{N}_{g}} \sum_{k \in \mathcal{K}} w_{n_{g}, k} M_{n_{g}, k}-c_{g}^{\mathrm{P}} \sum_{n_{g} \in \mathcal{N}_{g}} \sum_{k \in \mathcal{K}} w_{n_{g}, k} P_{n_{g}, k},
\end{aligned}
$$

where $c_{g}^{\mathrm{M}}$ and $c_{g}^{\mathrm{P}}$ are pricing factors for the number of allocated antennas and the transmit power of slice $g$, respectively. Hence, the resource provisioning problem can be written as

$$
\max _{\mathbf{P}, \mathbf{w}, \mathbf{M}} \sum_{g \in \mathcal{G}} U_{g}(\mathbf{P}, \mathbf{w}, \mathbf{M}),
$$

subject to the following constraints $\mathrm{C} 1-\mathrm{C} 4$.

C1: Exclusive sub-carrier allocation in OFDMA implies:

$w_{n_{g}, k} \in\{0,1\}$ and $\sum_{g \in \mathcal{G}} \sum_{n_{g} \in \mathcal{N}_{g}} w_{n_{g}, k} \leq 1, \quad \forall k \in \mathcal{K}$.
C2: Transmit power limitation for each user $n_{g}$ implies:

$$
\sum_{k \in \mathcal{K}} w_{n_{g}, k} P_{n_{g}, k} \leq P_{n_{g}}^{\max }, \quad \forall n_{g} \in \mathcal{N}_{g}, \quad \forall g \in \mathcal{G},
$$

where $P_{n_{g}}^{\max }$ is the maximum transmit power of user $n_{g}$. C3: Minimum required rate, $R_{g}^{\text {rsv }}$, of slice $g \in \mathcal{G}$ implies:

$$
\sum_{n_{g} \in \mathcal{N}_{g}} R_{n_{g}} \geq R_{g}^{\text {rsv }}, \quad \forall g \in \mathcal{G} .
$$

C4: Control on the number of antennas allocated to each slice to preserve the fairness between slices and to improve the energy efficiency for the WVNs can be expressed as [2],

$\sum_{n_{g} \in \mathcal{N}_{g}} \sum_{k \in \mathcal{K}} w_{n_{g}, k} M_{n_{g}, k} \in\left\{M_{g}^{\min }, M_{g}^{\min +1}, \cdots, M_{g}^{\max }\right\}$,

for each slice $g \in \mathcal{G}$ where $M_{g}^{\min }$ and $M_{g}^{\max }$ are the minimum number of reserved antennas and the maximum allowable number of allocated antennas for slice g, respectively.

Since (5) has combinatorial properties, it involves high computational complexity. Next, we propose an efficient algorithm to solve this problem by applying variable transformations and constraint relaxation.

\section{PRoposed AlgorithM}

To obtain a low-complexity solution to (5), we relax the conditions $\mathrm{C} 1, \mathrm{C} 3$, and $\mathrm{C} 4$, by considering $w_{n_{g}, k}$ as a continuous variable in the interval $[0,1]$ and the numbers of allocated antennas to be non-negative real-valued. In the new definition, $w_{n_{g}, k}$ indicates the fraction of time that sub-carrier $k$ is assigned to user $n_{g}$ for a specific transmission frame. Furthermore, we define two new variables: $x_{n_{g}, k}=w_{n_{g}, k} P_{n_{g}, k}$ and $y_{n_{g}, k}=w_{n_{g}, k} M_{n_{g}, k}$. For $M_{n_{g}, k} \gg 1, R_{n_{g}}$ can be approximated and represented as $\widetilde{R}_{n_{g}} \approx \sum_{k \in \mathcal{K}} w_{n_{g}, k} \log _{2}\left(\frac{x_{n_{g}, k} y_{n_{g}, k} d_{n_{g}}}{w_{n_{g}, k}^{2}}\right)$ for perfect CSI case or $\frac{T-\tau}{T} \sum_{k \in \mathcal{K}} w_{n_{g}, k} \log _{2}\left(\frac{\tau x_{n_{g}, k}^{2} y_{n_{g}, k} d_{n_{g}}^{2}}{w_{n_{g}, k}^{3}}\right)$ for imperfect CSI case, which are convex functions with respect to $x_{n_{g}, k}$, $y_{n_{g}, k}$, and $w_{n_{g}, k}$ [4], [5]. Consequently, conditions C1-C4 are changed to the following $\widetilde{\mathrm{C} 1}-\widetilde{\mathrm{C} 4}$, respectively,

$\widetilde{\mathrm{C} 1}: w_{n_{g}, k} \in[0,1]$ and $\sum_{g \in \mathcal{G}} \sum_{n_{g} \in \mathcal{N}_{g}} w_{n_{g}, k} \leq 1, \forall k \in \mathcal{K}$,

$\widetilde{\mathrm{C} 2}: \quad \sum_{k \in \mathcal{K}} x_{n_{g}, k} \leq P_{n_{g}}^{\max }, \quad \forall n_{g} \in \mathcal{N}_{g}, \quad \forall g \in \mathcal{G}$,

$\widetilde{\mathrm{C} 3}: \sum_{n_{g} \in \mathcal{N}_{g}} \widetilde{R}_{n_{g}} \geq R_{g}^{\mathrm{rsv}}, \quad \forall g \in \mathcal{G}$,

$\widetilde{\mathrm{C} 4}: M_{g}^{\min } \leq \sum_{n_{g} \in \mathcal{N}_{g}} \sum_{k \in \mathcal{K}} y_{n_{g}, k} \leq M_{g}^{\max }, \forall g \in \mathcal{G}$,

and (5) is transformed to

$$
\begin{array}{rc}
\max _{\mathbf{x}, \mathbf{w}, \mathbf{y}} & \sum_{g \in \mathcal{G}} \widetilde{U}_{g}(\mathbf{x}, \mathbf{w}, \mathbf{y}), \\
\text { subject to : } & \widetilde{\mathrm{C} 1}-\widetilde{\mathrm{C} 4},
\end{array}
$$

where $\mathbf{x}$ and $\mathbf{y}$ are vectors of all $x_{n_{g}, k}$ and $y_{n_{g}, k}$, and

$$
\begin{aligned}
& \widetilde{U}_{g}(\mathbf{x}, \mathbf{w}, \mathbf{y})=\sum_{n_{g} \in \mathcal{N}_{g}} \widetilde{R}_{n_{g}}(\mathbf{x}, \mathbf{w}, \mathbf{y})- \\
& \quad c_{g}^{\mathrm{M}} \sum_{n_{g} \in \mathcal{N}_{g}} \sum_{k \in \mathcal{K}} y_{n_{g}, k}-c_{g}^{\mathrm{P}} \sum_{n_{g} \in \mathcal{N}_{g}} \sum_{k \in \mathcal{K}} x_{n_{g}, k} .
\end{aligned}
$$


Since (6) involves continuous variables and convex functions, we can solve it with the following Lagrange function.

$$
\begin{aligned}
& \mathcal{L}\left(\mathbf{x}, \mathbf{w}, \mathbf{y}, \lambda_{n_{g}}, \phi_{g}, \theta_{g}, \psi_{g}\right)= \\
& -\sum_{g \in \mathcal{G}} \widetilde{U}_{g}+\sum_{n_{g} \in \mathcal{N}_{g}} \lambda_{n_{g}}\left(\sum_{k \in \mathcal{K}} x_{n_{g}, k}-P_{\max }\right) \\
& +\sum_{g \in \mathcal{G}} \phi_{g}\left(R_{g}^{\mathrm{rsv}}-\sum_{n_{g} \in \mathcal{N}_{g}} \widetilde{R}_{n_{g}}\right) \\
& +\sum_{g \in \mathcal{G}} \theta_{g}\left(M_{g}^{\mathrm{min}}-\sum_{n_{g} \in \mathcal{N}_{g}} \sum_{k \in \mathcal{K}} y_{n_{g}, k}\right) \\
& +\sum_{g \in \mathcal{G}} \psi_{g}\left(\sum_{n_{g} \in \mathcal{N}_{g}} \sum_{k \in \mathcal{K}} y_{n_{g}, k}-M_{g}^{\max }\right),
\end{aligned}
$$

where $\lambda_{n_{g}}, \phi_{g}, \theta_{g}$ and $\psi_{g}$ are the Lagrange multipliers for $\widetilde{\mathrm{C} 2}, \widetilde{\mathrm{C} 3}$ and $\widetilde{\mathrm{C}} 4$. Now, we propose an iterative approach to solve the dual problem of (7), in the following algorithm.

\section{Algorithm:}

\section{Initialization:}

Set $\mathbf{w}^{*}(l=0)=1, \mathbf{P}_{n_{g}}^{*}(l=0)=P_{n_{g}}^{\max } / K$, and $\mathbf{M}^{*}(l=$ $0)=M_{g}^{\max }$ for all $n_{g} \in \mathcal{N}_{g}$ and $g \in \mathcal{G}$. Initialize $l_{\max } \gg$

\section{Repeat:}

$1,0<\varepsilon \ll 1, \lambda(l=0), \boldsymbol{\psi}(l=0)$ and $\boldsymbol{\theta}(l=0)$.

- Update dual variables, $\lambda_{n_{g}}, \phi_{g}, \theta_{g}$ and $\psi_{g}$, by gradient descent method for all $g \in \mathcal{G}$ where $[x]^{+}=\max \{x, 0\}$ :

$$
\begin{aligned}
& \lambda_{n_{g}}(l+1)=\left[\lambda_{n_{g}}(l)+\delta_{\lambda_{n_{g}}} \frac{\partial \mathcal{L}}{\partial \lambda_{n_{g}}}\right]^{+}, \forall n_{g} \in \mathcal{N}, \\
& \phi_{g}(l+1)=\left[\phi_{g}(l)+\delta_{\phi_{g}} \frac{\partial \mathcal{L}}{\partial \phi_{g}}\right]^{+}, \quad \forall g \in \mathcal{G}, \\
& \theta_{g}(l+1)=\left[\theta_{g}(l)+\delta_{\theta_{g}} \frac{\partial \mathcal{L}}{\partial \theta_{g}}\right]^{+}, \quad \forall g \in \mathcal{G}, \\
& \psi_{g}(l+1)=\left[\psi_{g}(l)+\delta_{\psi_{g}} \frac{\partial \mathcal{L}}{\partial \psi_{g}}\right]^{+}, \quad \forall g \in \mathcal{G} .
\end{aligned}
$$

- Using the above updated parameters for $(l+1)$, compute $P_{n_{g}, k}^{*}(l+1)$ and $M_{n_{g}, k}^{*}(l+1)$ for all $n_{g} \in \mathcal{N}_{g}$, and $k \in \mathcal{K}$.

\begin{tabular}{|l|l|l|}
\hline $\begin{array}{l}\text { For } \\
l+1\end{array}$ & Perfect CSI case & Imperfect CSI case \\
\hline \hline$P_{n_{g}, k}^{*}$ & {$\left[\frac{1+\phi_{g}}{\ln (2)\left(\lambda_{n_{g}}+c_{g}^{P}\right)}\right]_{0}^{P_{n_{g}}^{\max }}$} & $\frac{(T-\tau)}{T}\left[\frac{2\left(1+\phi_{g}\right)}{\ln (2)\left(\lambda_{n_{g}}+c_{g}^{P}\right)}\right]_{0}^{P_{n_{g}}^{\max }}$ \\
\hline$M_{n_{g}, k}^{*}$ & {$\left[\frac{1+\phi_{g}}{\ln (2)\left(\psi_{g}-\theta_{g}+c_{g}^{M}\right)}\right]_{0}^{M_{g}^{\max }}$} & $\frac{(T-\tau)}{T}\left[\frac{1+\phi_{g}}{\ln (2)\left(\psi_{g}-\theta_{g}+c_{g}^{M}\right)}\right]_{0}^{M_{g}^{\max }}$ \\
\hline
\end{tabular}

where $[x]_{a}^{b}=\min \{b, \max \{x, a\}\}$.

- Perform sub-carrier allocation for all $n_{g} \in \mathcal{N}_{g}$ and for all $k \in \mathcal{K}$, where by applying KKT conditions [6], [7],

$\frac{\partial \mathcal{L}}{\partial w_{n_{g}, k}}=\left(1+\phi_{g}\right) \times$

$\left\{\begin{array}{l}\log _{2}\left(P_{n_{g}, k} d_{n_{g}} M_{n_{g}, k}\right)-\frac{2}{\ln (2)}, \text { perfect CSI } \\ \frac{(T-\tau)}{T}\left(\log _{2}\left(\tau P_{n_{g}, k}^{2} d_{n_{g}}^{2} M_{n_{g}, k}\right)-\frac{3}{\ln (2)}\right), \text { imperfect CSI, }\end{array}\right.$

and consequently, the optimal sub-carrier allocation $w_{n_{g}, k}^{*}(l+1)$ can be derived as in [4], i.e., $w_{n_{g}, k}^{*}(l+1)=$ 1 , if $\frac{\partial \mathcal{L}}{\partial w_{n_{g}, k}}>0, w_{n_{g}, k}^{*}(l+1)=0$, otherwise.

- Set $l=l+1$

Until $\|\mathbf{P}(l+1)-\mathbf{P}(l)\| \leq \varepsilon$ or $l \geq l_{\max }$.
Finally, the optimum integer $M_{n_{g}, k}^{*}$ is selected as $\left\lfloor M_{n_{g}, k}(l)\right\rfloor$ where $\lfloor x\rfloor$ denotes the largest integer less than or equal to the value of $x$.

\section{Simulation Results}

To evaluate the performance of WVN with the proposed algorithm, we consider one BS serving two slices (i.e., $G=$ 2) with $N_{1}=N_{2}=4$. Furthermore, we assume $K=64$ and $P_{n_{g}}^{\max }=0 \mathrm{~dB}$. After precoding, channel is modelled as $\left\|h_{n_{g}, k, m_{n_{g}, k}}\right\|^{2}=d_{n_{g}}=D_{n_{g}}^{-\beta}$ where $\beta=3$ is the path loss exponent and $D_{n_{g}} \in[0.2,0.6]$ is the distance of user $n_{g}$ to BS. Furthermore, we set $\varepsilon=10^{-4}$ for Algorithm 1 and $R_{1}^{\text {rsv }}=R_{2}^{\text {rsv }}=2 \mathrm{bps} / \mathrm{Hz}$ unless otherwise stated. $T$ is selected based on the parameters in [3] and simulations are based on the ratio of $\tau / T$. All the results are presented in terms of average rate over 100 channel realizations based on random locations of the users. When $R_{g}^{\text {rsv }}$ does not hold for a channel realization, the total rate is set to zero. Also, to demonstrate the effect of $c_{g}^{\mathrm{M}}$ and $c_{g}^{\mathrm{P}}$, we consider three sets with different values of $c_{g}^{\mathrm{M}}$ and $c_{g}^{\mathrm{P}}$. For Set 1 , we have $c_{g}^{\mathrm{M}}=c_{g}^{\mathrm{P}}=0$ which means that the utility of each user is equal to its rate. For Set 2, we have $c_{g}^{\mathrm{M}}=0.07$ and $c_{g}^{\mathrm{P}}=1$. Set 3 imposes higher restrictions to use antennas and power by employing $c_{g}^{\mathrm{M}}=0.09$ and $c_{g}^{\mathrm{P}}=2$.

Fig. 1 shows that the total rate is a non-decreasing function of $M_{g}^{\max }$ in both perfect and imperfect CSI cases where $\frac{\tau}{T}=0.3$. This can be explained by the fact that the feasibility regions are expanded as $M_{g}^{\max }$ increases. However the utility function is formulated as a decreasing function of $c_{g}^{\mathrm{P}}$ and $c_{g}^{\mathrm{M}}$, so that by increasing the costs from Set 1 to Set 3, the total rate of $\mathrm{WVN}$ is decreased. As a result, the increase in the achieved rate is reduced for large values of $M_{g}^{\max }$, especially for high $c_{g}^{P}$ and/or $c_{g}^{M}$, e.g., for $M_{g}^{\max }>75$, the total rate for Sets 2 and 3 is almost unchanged. Furthermore, due to the channel estimation errors, the performance with imperfect CSI is worse than that with perfect CSI for all sets, as expected.

To demonstrate the effects of $R_{g}^{\text {rsv }}$, Fig. 2 plots the total rate versus $R_{g}^{\text {rsv }}$ for Set 1 , where $\frac{\tau}{T}=0.3, M_{1}^{\max }=M_{2}^{\max }=$ 100 , and $R_{1}^{\text {rsv }}=R_{2}^{\text {rsv }}=R_{g}^{\text {rsv }}$. It can be observed that in both perfect and imperfect CSI cases, the total rate decreases with increasing $R_{g}^{\text {rsv }}$. This is because increasing $R_{g}^{\text {rsv }}$ shrinks the feasibility region for convex optimization problem (5) and thus reduces the optimal value of objective function [5].

To study further the effect of $\tau$ on the performance of WVN, we focus on Set 1 with $M_{g}^{\max }=\{200,150\}$ and plot the rate versus $\frac{\tau}{T}$ in Fig. 3. Obviously, when $0.1 \leq \frac{\tau}{T} \leq 0.3$, the rate increases with increasing $\frac{\tau}{T}$ benefiting from the more accurate CSI estimation. However, for $0.3 \leq \frac{\tau}{T} \leq 0.9$, the spectral efficiency of WVN is decreased with increasing $\frac{\tau}{T}$ due to the fact that most of the transmission time wastes for pilot signals. Therefore, considering the optimum values of $\frac{\tau}{T}$ to reach the best performance of WVN is essential. Fig. 3 also highlights that increasing $M_{g}^{\max }$ can increase the total rate for any $\tau$ due to the feasibility region expansion.

To further study the effects of pricing on the total rate of WVN, in Fig. 4, the total rate is plotted versus $c_{g}^{\mathrm{M}}$ and $c_{g}^{\mathrm{P}}$. Obviously, the total rate is decreased with increasing $c_{g}^{\mathrm{M}}$ 


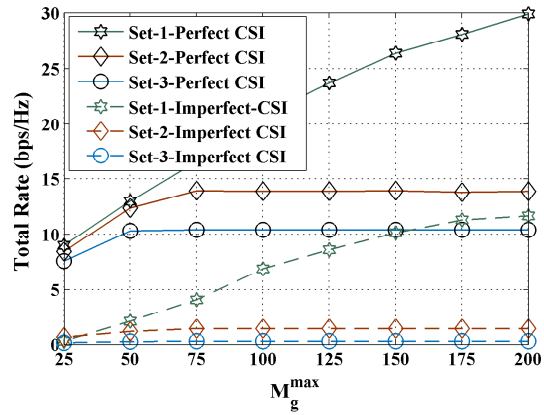

Fig. 1: Total rate vs. $M_{g}^{\max }$

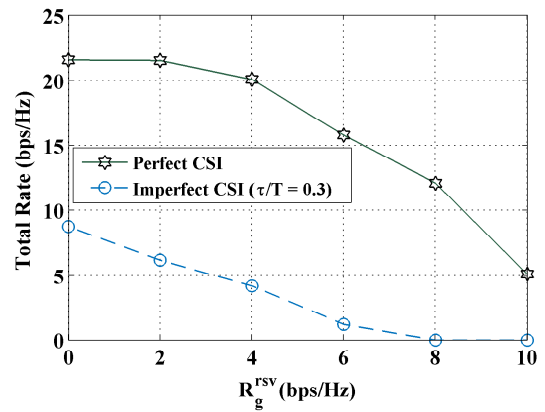

Fig. 2: Total rate vs. $R_{g}^{\text {rsv }}$

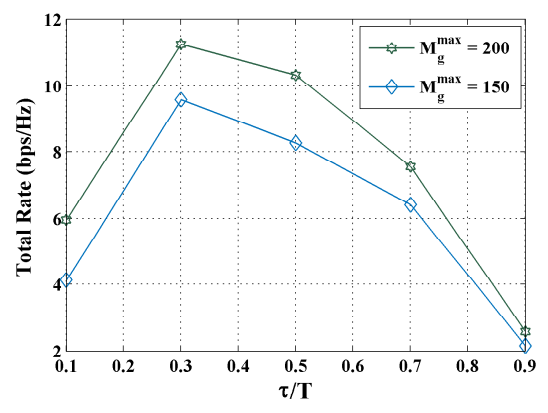

Fig. 3: Total rate vs. $\tau / T$

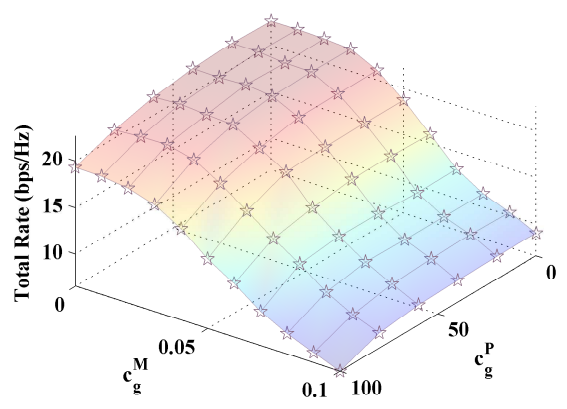

Fig. 4: Total Rate vs. $c_{g}^{\mathrm{P}}$ and $c_{g}^{\mathrm{M}}$

and $c_{g}^{\mathrm{P}}$. However, the effect of increasing $c_{g}^{\mathrm{M}}$ is much more profound compared to that of $c_{g}^{\mathrm{P}}$. This is because in massive MIMO, we have $M_{g}^{\max } \gg P_{n_{g}}^{\max }$, e.g., compare $M_{g}^{\max }>100$ and $P_{n_{g}}^{\max }=0 \mathrm{~dB}$. Figures 3 and 4 highlight that allocating dynamic and optimal pricing values for WVN and optimum value of $\tau / T$ are essential to reach the best performance which are left for the future work.

\section{CONCLUSion}

Utility-based resource provisioning for massive-MIMObased WVN was investigated in this letter for perfect and imperfect CSI scenarios. We propose an efficient iterative algorithm to solve the developed resource allocation problems. Via simulation results, the effects of power and antenna pricing mechanisms on the performance of WVN were investigated.

VI. ApPENDiX: DeRIVATION OF USER RATE $R_{n_{g}, k}$ IN (3)

For mutually independent $1 \times n$ vectors $\mathbf{p}=\left[p_{1}, \cdots p_{n}\right]$ and $\mathbf{q}=\left[q_{1}, \cdots q_{n}\right]$ whose elements are i.i.d. zero-mean, unity-variance random variables (RVs), it can be shown by the law of large numbers that $\lim _{n \rightarrow \infty} \frac{1}{n} \mathbf{p p}^{\mathrm{H}} \stackrel{\text { a.s. }}{\longrightarrow} 1$ and $\lim _{n \rightarrow \infty} \frac{1}{n} \mathbf{p q}^{\mathrm{H}} \stackrel{\text { a.s. }}{\longrightarrow} 0$ where $\stackrel{\text { a.s. }}{\longrightarrow}$ denotes the almost sure convergence.

For perfect CSI using MRC, $\mathbf{f}_{n_{g}, k}=\mathbf{h}_{n_{g}, k}^{\mathrm{H}}$ [3], and the SINR of user $n_{g}$ on sub-carrier $k$ is $\gamma_{n_{g}, k}^{\text {Perf }}=$ $P_{n_{g}, k}\left\|\mathbf{h}_{n_{g}, k}\right\|^{4} /\left(\left\|I_{n_{g}, k}\right\|^{2}+\left\|\mathbf{h}_{n_{g}, k}\right\|^{2}\right)$. According to the law of large numbers for large $M_{n_{g}, k},\left\|\mathbf{h}_{n_{g}, k}\right\|^{2} \rightarrow M_{n_{g}, k} d_{n_{g}}$ and $\left\|I_{n_{g}, k}\right\|^{2} \rightarrow 0$. Thus, $R_{n_{g}, k}^{\text {Perf }}=\log _{2}\left(1+P_{n_{g}, k} d_{n_{g}} M_{n_{g}, k}\right)$ as shown in (3).

For imperfect CSI in (2),

$$
\delta_{n_{g}, k}(\mathbf{P})=\sum_{g \in \mathcal{G}} \sum_{n_{g}^{\prime} \neq n_{g}} \sqrt{P_{n_{g}^{\prime}, k}} \widetilde{\mathbf{h}}_{n_{g}^{\prime}, k} \mathbf{f}_{n_{g}, k}^{\text {Imperf }} x_{m_{g}, k}
$$

$$
-\sqrt{P_{n_{g}, k}} \sum_{g \in \mathcal{G}} \sum_{n_{g}^{\prime} \in \mathcal{N}_{g}} \mathbf{e}_{n_{g}^{\prime}, k} \mathbf{f}_{n_{g}, k}^{\text {Imperf }} x_{m_{g}, k}+\mathbf{z}_{n_{g}, k} \mathbf{f}_{n_{g}, k}^{\text {Imperf }}
$$

where $\mathbf{e}_{n_{g}, k}=\mathbf{h}_{n_{g}, k}-\widetilde{\mathbf{h}}_{n_{g}, k}$ whose elements are RVs with zero mean and variance $\frac{P_{n_{g}, k}^{\text {Pilot }} d_{n_{g}}}{P_{n_{g}, k}^{\text {Pilot }} d_{n_{g}}+1}$ and $P_{n_{g}, k}^{\text {Pilot }}=\tau P_{n_{g}, k}$ [3]. With MMSE-based channel estimation, the elements of $\widetilde{\mathbf{h}}_{n_{g}, k}$ are i.i.d. RVs with zero mean and variance $\frac{P_{n_{g}, k}^{\text {Pilot }} d_{n_{g}}^{2}}{P_{n_{g}, k}^{\text {Pilot }} d_{n_{g}}+1}$ [3]. With MRC precoder, $\mathbf{f}_{n_{g}, k}^{\text {Imperf }}=\widetilde{\mathbf{h}}_{n_{g}, k}^{\mathrm{H}}$. Since $\mathbf{e}_{n_{g}^{\prime}, k}$ and $\mathbf{h}_{n_{g}^{\prime}, k}$ are independent of $\widetilde{\mathbf{h}}_{n_{g}, k}$ and $\mathbf{f}_{n_{g}, k}^{\text {Imperf }}$, the first term of $\delta_{n_{g}, k}(\mathbf{P})$ is zero and second term is equal to $\sqrt{P_{n_{g}, k}} \mathbf{e}_{n_{g}, k} \mathbf{f}_{n_{g}, k}^{\text {Imperf }} x_{n_{g}, k}$. Thus, the SINR for the imperfect CSI case is $\gamma_{n_{g}, k}^{\text {Imperf }}=P_{n_{g}, k}\left\|\widetilde{\mathbf{h}}_{n_{g}, k}\right\|^{4} /\left(P_{n_{g}, k}\left\|\mathbf{e}_{n_{g}, k} \widetilde{\mathbf{h}}_{n_{g}, k}^{\mathrm{H}}\right\|^{2}+\left\|\widetilde{\mathbf{h}}_{n_{g}, k}\right\|^{2}\right)$

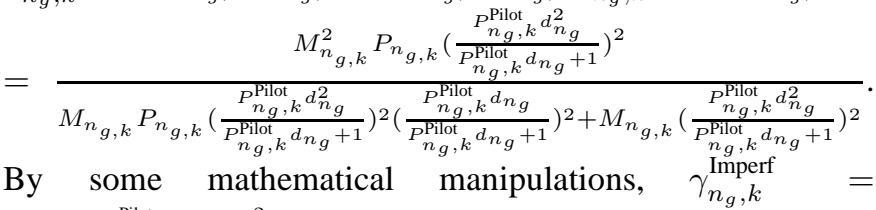
$\frac{M_{n_{g}, k} P_{n_{g}, k}^{\text {Pilot }} P_{n_{g}, k} d_{n_{g}}^{2}}{P_{n_{g}, k} d_{n_{g}}+P_{n_{g}, k}^{\text {Pilot }} d_{n_{g}}+1}$. Now, by substituting $P_{n_{g}, k}^{\text {Pilot }}=\tau P_{n_{g}, k}$ and considering $P_{n_{g}, k}=\rho_{n_{g}, k} / \sqrt{M_{n_{g}, k}}$ [3], we have $\gamma_{n_{g}, k}^{\text {Imperf }}=\frac{\tau \rho_{n_{g}, k}^{2} d_{n_{g}}^{2}}{1+(1+\tau) \rho_{n_{g}, k} d_{n_{g}} / \sqrt{M_{n_{g}, k}}}$. When $M_{n_{g}, k} \rightarrow \infty$, we have $\gamma_{n_{g}, k}^{\text {Imperf }}=\tau \rho_{n_{g}, k}^{2} d_{n_{g}}^{2}=\tau P_{n_{g}, k}^{2} d_{n_{g}}^{2} M_{n_{g}}$. Thus, as shown in (3), $R_{n_{g}, k}=\frac{T-\tau}{T} \log _{2}\left(1+\tau P_{n_{g}, k}^{2} d_{n_{g}}^{2} M_{n_{g}}\right)$ where $(T-\tau) / T$ is the fraction of transmission frame for sending the actual data [3].

\section{REFERENCES}

[1] R. Kokku et al., "NVS: A substrate for virtualizing wireless resources in cellular networks," IEEE/ACM Trans. Netw., vol. 20, no. 5, Oct. 2012.

[2] L. Lu et al., "An overview of massive MIMO: Benefits and challenges," IEEE J. Sel. Topics Signal Process., vol. 8, no. 5, pp. 742-758, Oct. 2014.

[3] H. Q. Ngo, E. Larsson, and T. Marzetta, "Energy and spectral efficiency of very large multiuser MIMO systems," IEEE Trans. Commun., vol. 61, no. 4, pp. 1436-1449, April 2013.

[4] D. Ng, E. Lo, and R. Schober, "Energy-efficient resource allocation in OFDMA systems with large numbers of base station antennas," IEEE Trans. Wireless Commun., vol. 11, no. 9, pp. 3292-3304, September 2012.

[5] S. Boyd and L. Vandenberghe, Convex optimization. Cambridge, 2009.

[6] M. Tao, Y.-C. Liang, and F. Zhang, "Resource allocation for delay differentiated traffic in multiuser OFDM systems," IEEE Trans. Wireless Commun., vol. 7, no. 6, pp. 2190-2201, June 2008.

[7] H. Zhang et al., "Resource allocation in spectrum-sharing OFDMA femtocells with heterogeneous services," IEEE Trans. Commun., vol. 62, no. 7, pp. 2366-2377, July 2014. 